\title{
Gephyrin Oligomerization Controls GlyR Mobility and Synaptic Clustering
}

\author{
Martino Calamai, ${ }^{1,2 \star}$ Christian G. Specht, ${ }^{1,2 *}$ Janosch Heller, ${ }^{2,3}$ Damien Alcor, ${ }^{1,2}$ Patricia Machado, ${ }^{1,2}$ \\ Christian Vannier, ${ }^{1,2}$ and Antoine Triller ${ }^{1,2}$ \\ ${ }^{1}$ INSERM U789 -Biologie Cellulaire de la Synapse and 2Département de Biologie, Ecole Normale Supérieure, 75005 Paris, France, and ${ }^{3}$ Institute of \\ Biochemistry, University of Cologne, D-50674 Cologne, Germany
}

\begin{abstract}
High local concentrations of glycine receptors (GlyRs) at inhibitory postsynaptic sites are achieved through their binding to the scaffold protein gephyrin. The $\mathrm{N}$ - and C-terminal domains of gephyrin are believed to trimerize and dimerize, respectively, thus contributing to the formation of submembranous gephyrin clusters at synapses. GlyRs are associated with gephyrin also at extrasynaptic locations. We have investigated how gephyrin oligomerization influences GlyR dynamics and clustering in COS-7 cells and in cultured spinal cord neurons. To this aim, we have expressed isolated $\mathrm{N}$ - and C-terminal domains of gephyrin that interfere with the oligomerization of the full-length protein. We also studied the effect of an endogenous splice variant, ge $(2,4,5)$, with a decreased propensity to trimerize. A reduction of the size and number of gephyrin-GlyR clusters was found in cells expressing the various interfering gephyrin constructs. Using fluorescence recovery after photobleaching, we studied the exchange kinetics of synaptic gephyrin clusters. Real-time singleparticle tracking was used to analyze the mobility of GlyRs. We found that all the tested constructs displayed faster rates of recovery than wild-type gephyrin and increased the mobility of extrasynaptic receptors, showing that gephyrin-gephyrin interactions modulate the lateral diffusion of GlyRs. Furthermore, we observed an inverse correlation between GlyR diffusion properties and gephyrin cluster size that depended on the number of binding sites blocked by the different constructs. Since alterations in the oligomerization properties of gephyrin are related to the dynamics of GlyRs, the gephyrin splice variant ge $(2,4,5)$ may be implicated in the modulation of synaptic strength.
\end{abstract}

\section{Introduction}

Lateral diffusion and local trapping are key parameters in the regulation of neurotransmitter receptor numbers at synapses and thus for the modulation of synaptic strength (for review, see Triller and Choquet, 2008). Interactions of receptors with cytoplasmic scaffold proteins are responsible for the confinement and accumulation of receptors in front of presynaptic release sites (for review, see Specht and Triller, 2008). Among these proteins, gephyrin is the core molecule for anchoring and stabilizing glycine and $\mathrm{GABA}_{\mathrm{A}}$ receptors $\left(\mathrm{GlyR}\right.$ and $\left.\mathrm{GABA}_{\mathrm{A}} \mathrm{R}\right)$ at inhibitory postsynaptic sites (Kirsch et al., 1993; Meier et al., 2001; Fritschy et al., 2008; Tretter et al., 2008). The dynamic regulation of GlyR clustering is not based exclusively on the association between the receptor and gephyrin, since at least $40 \%$ of GlyRs diffusing outside of synapses are bound to gephyrin (Ehrensperger et al., 2007). Therefore, in addition to gephyrin-GlyR interactions, gephyrin-gephyrin interactions also have to be taken into account. Several recent studies have begun to explore the role of

Received 0ct. 13, 2008; revised April 1, 2009; accepted April 23, 2009

This work was supported by the Federation of European Biochemical Societies, the Association Française contre les Myopathies, and the Institut de Recherche sur la Moelle Epinière. We thankC. Charrier and C. Ribrault for technical assistance.

*M.C. and C.G.S. contributed equally to this work.

Correspondence should be addressed to Antoine Triller, INSERM U789-Biologie Cellulaire de la Synapse, Département de Biologie, Ecole Normale Supérieure, 46, rue d’Ulm, 75005 Paris, France. E-mail: triller@biologie.ens.fr. DOI:10.1523/JNEUROSCI.5711-08.2009

Copyright $\odot 2009$ Society for Neuroscience $\quad$ 0270-6474/09/297639-10\$15.00/0 gephyrin oligomerization in GlyR and $\mathrm{GABA}_{\mathrm{A}} \mathrm{R}$ clustering (Maas et al., 2006; Lardi-Studler et al., 2007; Saiyed et al., 2007).

The gephyrin sequence contains an $\mathrm{N}$ - and a C-terminal domain ( $\mathrm{G}$ and $\mathrm{E}$ ), connected by a central linker region (C) (for review, see Fritschy et al., 2008). The full-length protein can form trimers, hexamers, and higher-order structures (Bedet et al., 2006; Saiyed et al., 2007), whereas isolated G and E domains trimerize (Schwarz et al., 2001) or dimerize (Sola et al., 2004; Kim et al., 2006), respectively. It has, therefore, been postulated that gephyrin may form a submembranous hexagonal lattice (Kneussel and Betz, 2000; Xiang et al., 2001; Bedet et al., 2006). However, the oligomerization mechanism of gephyrin is not straightforward, since the dimerization of the full-length protein by the $\mathrm{E}$ domain is inhibited by the $\mathrm{C}$ domain (Bedet et al., 2006), whereas it may be favored by GlyR binding (Kim et al., 2006).

Various splice variants of gephyrin have been characterized in human and rodent cells (Prior et al., 1992; Ramming et al., 2000; David-Watine, 2001; Paarmann et al., 2006a,b). A full-length gephyrin splice variant $[\mathrm{ge}(2,4,5)]$ with a decreased trimerization potential has been used to study gephyrin oligomerization. Insertion of cassette 5 in the G domain (or G2) (for nomenclature, see Fritschy et al., 2008) is responsible for the lack of trimerization of ge $(2,4,5)$ (Bedet et al., 2006; Saiyed et al., 2007). Furthermore, over-expression of ge $(2,4,5)$ favors the loss of both GlyR and gephyrin clusters in spinal cord neurons (Bedet et al., 2006). The mechanisms responsible for the assembly or disassembly of 
gephyrin clusters and the regulation of GlyR diffusion in the presence of ge $(2,4,5)$, however, remain elusive.

To address these issues, we investigated the diffusion dynamics of GlyRs while interfering with gephyrin oligomerization. We found that expression of the isolated $\mathrm{G}$ and $\mathrm{E}$ domains interfered with gephyrin oligomerization and caused an increase in the mobility of extrasynaptic gephyrin-GlyR complexes. Furthermore, over-expression of the ge $(2,4,5)$ variant resulted in a similar increase in the lateral diffusion of GlyRs, in addition to a decrease in synaptic gephyrin clustering. This result directly relates the reduction of synaptic clusters to the formation of extrasynaptic GlyR-ge $(2,4,5)$ complexes.

\section{Materials and Methods}

Gephyrin constructs. $\mathrm{Ge}(2)$ and ge $(2,4,5)$ clones have been described previously (Meier et al., 2000b). Ge(2) corresponds to clone P1 (Prior et al., 1992). The construction of chimeric $\mathrm{GlyR}_{\alpha 1} \beta g \mathrm{~g}$ subunit, vege(2), mrfpge(2), vege $(2,4,5)$, and $\operatorname{mrfp}(2,4,5)$ have been described previously (Meier et al., 2000b; Bedet et al., 2006). PCR fragments containing the $\mathrm{G}(2), E$, and $\mathrm{G}(2,5)$ sequences were generated using the $\mathrm{pC}$-vege $(2)$ and pC-vege $(2,4,5)$ plasmids as templates. The PCR fragments were shuttled into $\mathrm{pC}$-vege(2) and $\mathrm{pC}$-mrfpge(2) using the HindIII/NotI restriction sites, replacing the full-length sequence in these constructs (Bedet et al., 2006). Isolated $\mathrm{G}(2), \mathrm{G}(2,5)$, and $\mathrm{E}$ domains encompass residues 1 to 183 , 1 to 196 , and 318 to 736 of the corresponding full-length proteins, respectively.

Cell culture and transfection. African green monkey kidney (COS-7) cells were grown in MEM (Invitrogen) containing 10\% fetal calf serum (FCS) at $37^{\circ} \mathrm{C}$ and $5 \% \mathrm{CO}_{2}$. Subconfluent cultures were transfected using FuGENE 6 (Roche Applied Science) with a total of $2 \mu \mathrm{g}$ plasmid DNA. $\operatorname{GlyR}_{\alpha 1} \beta \mathrm{gb}$, vege(2), and monomeric fluorescent protein (mrfp)-tagged plasmids were cotransfected in a stoichiometry of 2:1:1, respectively. After transfection, the medium was replaced with MEM containing $2 \%$ FCS and COS-7 cells expressed the recombinant protein for up to $24 \mathrm{~h}$.

Spinal cord neurons were prepared from embryonic (E14) Sprague Dawley rats as described previously (Meier et al., 2000a). Briefly, cells were grown in neurobasal medium supplemented with B27, 2 mM glutamine, and antibiotics (Invitrogen) at $36^{\circ} \mathrm{C}$ and $5 \% \mathrm{CO}_{2}$. Neurons were transfected 8-9 d after plating using Lipofectamine2000 (Invitrogen) with $2 \mu \mathrm{g}$ of plasmid DNA. Protein expression was allowed to proceed for 24 to $48 \mathrm{~h}$. Vege (2) and mrfp-tagged constructs were used in a stoichiometry of 1:1 for cluster size quantification and fluorescence recovery after photobleaching (FRAP) experiments. Only Venus-tagged constructs were transfected for single-particle tracking (SPT) experiments. Spinal cord primary neurons from homozygous mrfp-gephyrin knock-in mice (P. Machado and A. Triller, unpublished observations) were prepared at E13 and cultured as described above.

Immunofluorescence surface labeling of fixed cells. To prevent nonspecific labeling, living COS-7 cells were first incubated at $4^{\circ} \mathrm{C}$ in PBS containing $0.8 \mathrm{~mm} \mathrm{CaCl}_{2}, 0.5 \mathrm{~mm} \mathrm{MgCl}_{2}, 0.12 \%$ (w/v) gelatin, and $0.1 \%$ (w/v) BSA, as described previously (Rosenberg et al., 2001). Cell surface $\mathrm{GlyR}_{\alpha 1} \beta \mathrm{gb}$ subunits were labeled for $30 \mathrm{~min}$ with primary anti-c-myc monoclonal antibody (clone 9E10; Roche Applied Science) at a dilution of 1:400 in blocking solution. After several washes in PBS containing 0.8 $\mathrm{mm} \mathrm{CaCl} 2$ and $0.5 \mathrm{mM} \mathrm{MgCl}_{2}$, COS-7 cells were fixed in $4 \%(\mathrm{w} / \mathrm{v})$ paraformaldehyde in PBS for $15 \mathrm{~min}$, rinsed with PBS, and quenched with 50 $\mathrm{mM} \mathrm{NH}_{4} \mathrm{Cl}$ in PBS for $30 \mathrm{~min}$. After a brief wash with PBS, cells were incubated for $40 \mathrm{~min}$ with Cy5-conjugated goat anti-mouse (The Jackson Laboratory) at a 1:500 dilution. After two washes with PBS containing $0.12 \%$ gelatin and two with PBS, cells were mounted in Vectashield (Vector Laboratories).

Quantitative analysis of gephyrin clusters. Images of fixed COS-7 cells were acquired using a Leica Digital-Modul-R epifluorescence microscope $(100 \times / 1.4$ oil-immersion objective $)$ equipped with a chargecoupled device camera (Micromax; Princeton Instruments) and appropriate filters. Images of live neurons were acquired using the same microscope setup used for FRAP experiments (see below). Gephyrin clusters in COS-7 cells or neurons from at least two independent cultures were quantified using a multidimensional image analysis (MIA) (V. Racine, J. Salamero, and J. B. Sibarita, unpublished observations) interface running in MetaMorph software (Molecular Devices; Roper Scientific). Briefly, fluorescent clusters were detected with a procedure based on wavelet decomposition, allowing the selection of small structures with low intensities and large structures with high intensities at the same time (Théry et al., 2005).

The determination of the percentage of vege(2) clusters that colocalize with FM4-64-positive presynaptic terminal in $8-10 \mathrm{~d}$ in vitro (DIV) neurons was performed on regions of interest that included the dendrites and excluded the cell body. Images were thresholded with ImageJ software, creating binary masks corresponding to gephyrin or FM4-64 puncta. The percentage of clusters colocalizing with presynaptic terminals was then calculated by dividing the number of thresholded vege(2) clusters overlapping with FM4-64 puncta by the total number of vege(2) clusters. The analysis includes 13 cells from two independent experiments.

FRAP. Neurons were imaged in a heating chamber containing airequilibrated phenol red-free MEM medium supplemented with $33 \mathrm{~mm}$ glucose, 20 mM HEPES, 2 mm glutamine, 1 mm sodium pyruvate, and B27 at $37^{\circ} \mathrm{C}$. Photobleaching was performed using a Nikon TE2000-PFS microscope (100× oil-immersion objective) equipped with a FRAP-3D system (Photometrics; Roper Scientific) and QuantEM EMCCD camera (Photometrics; Roper Scientific). Using MetaMorph software, up to four regions of interest of $\sim 1.3 \mu \mathrm{m}^{2}$ containing single vege(2) clusters on dendrites were selected for FRAP. Bleaching was achieved using the 488 $\mathrm{nm}$ line of a $50 \mathrm{~mW}$ Ar laser for $10 \mathrm{~ms}$. Recovery was followed by timelapse imaging for $30 \mathrm{~min}$ using appropriate emission filters. At each time point, an image stack over $1.6 \mu \mathrm{m}$ was taken along the $z$-axis. Experiments were analyzed with ImageJ using a homemade macro developed by Xavier Darzacq. Briefly, the macro creates a maximum-projection of each stack, tracks the bleached spots, and measures their fluorescence recovery over time. The intensity values were then corrected for background fluorescence and for photobleaching attributable to image acquisition. Data were further normalized to the prebleaching fluorescence intensity and to the first time point after bleaching $\left(t_{0}=0\right)$, according to the following equations:

$$
\begin{gathered}
F_{\mathrm{t}}=\frac{I_{\mathrm{t}} \cdot I_{\mathrm{nb}, \mathrm{pre}}}{I_{\mathrm{pre}} \cdot I_{\mathrm{nb}, \mathrm{t}}}, \\
F_{\text {norm }}=\frac{F_{\mathrm{t}}-F_{0}}{1-F_{0}},
\end{gathered}
$$

where $F_{\mathrm{t}}$ is the relative fluorescence intensity recorded as a function of time; $I_{\mathrm{t}}$ and $I_{\mathrm{nb}, \mathrm{t}}$ are the intensities at time $t$ of the bleached and nonbleached spots, respectively; $I_{\text {pre }}$ and $I_{\mathrm{nb} \text {,pre }}$ are the raw intensities of the bleached and nonbleached spots, before beaching; $F_{\text {norm }}$ is the fluorescence at time $t$ normalized to the fluorescence intensity at time $t_{0}=0, F_{0}$. More than 15 clusters from at least five neurons from three independent experiments were analyzed for each construct.

For the mrfp-tagged constructs, background intensity was not subtracted from the data because of the low signal-to-noise ratio and the exceedingly fast recovery of some constructs. Furthermore, these data were all normalized to the intensity of $\operatorname{mrfpge}(2)$ at $t_{0}=0$, since some of the constructs displayed exceedingly fast recoveries.

Single-particle imaging. Quantum dots (QDs) labeling and live imaging have been extensively described (Bannai et al., 2006). Briefly, living cells were washed and incubated at $37^{\circ} \mathrm{C}$ with primary and secondary antibodies in air-equilibrated MEM (see above, FRAP), followed by QDs in QD-binding buffer. Neurons were further incubated with FM4-64 in $40 \mathrm{~mm} \mathrm{KCl}$ for $30 \mathrm{~s}$. Mouse anti-c-myc monoclonal antibody (1:40000 dilution) and mouse anti-GlyR $\alpha 1$ subunit (mAb2b, 1:600 dilution; Alexis Biochemicals) were used as primary antibodies in COS-7 cells and neurons, respectively. Biotinylated anti-mouse Fab antibody (The Jackson Laboratory) was used as secondary antibody at a 1:400 dilution. Streptavidin-coated QDs (Invitrogen) emitting at $605 \mathrm{~nm}$ were used at a 1:1000 dilution. Cells were monitored with an Olympus inverted microscope (IX71) equipped with an oil-immersion objective (Olympus times 
A

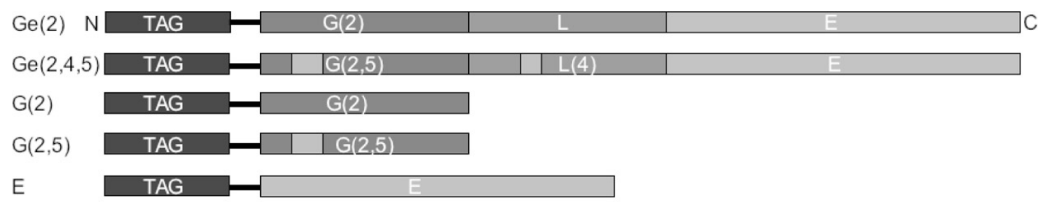

B
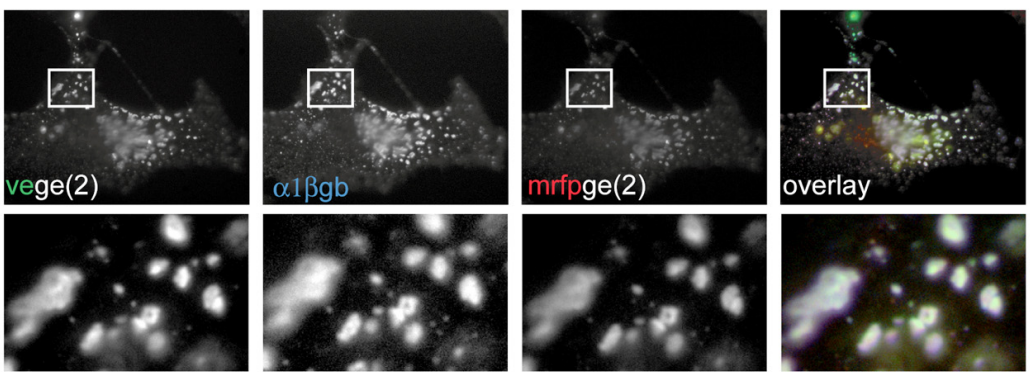

C
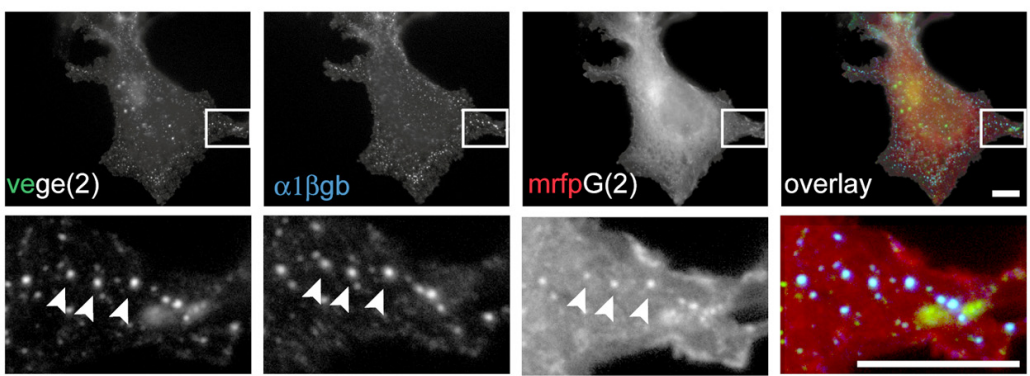

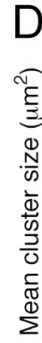

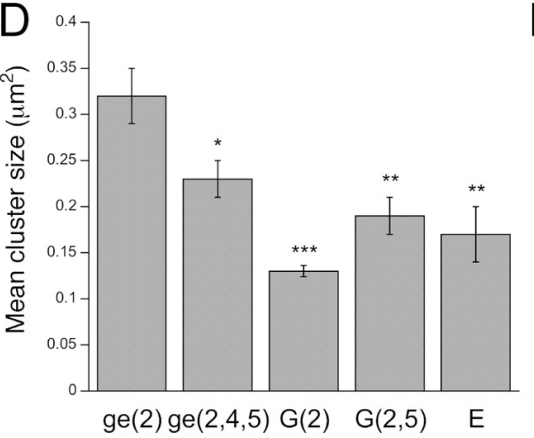

E

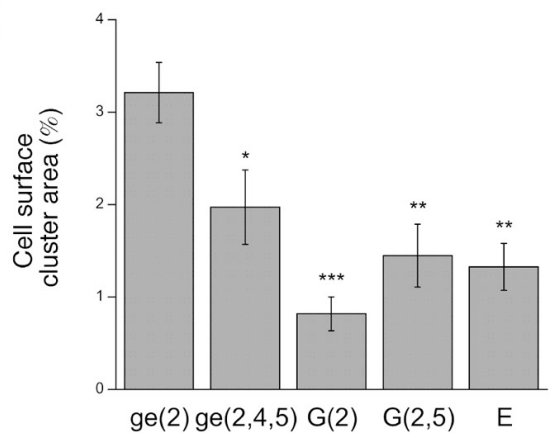

Figure 1. Structure and expression pattern of gephyrin constructs in $\operatorname{COS}-7$ cells. $A, G, C$ (or $\mathrm{L}$ ), and $\mathrm{E}$ correspond to the $\mathrm{N}$-terminal, central (or linker), and C-terminal domains of gephyrin, respectively. $\mathrm{Ge}(2)$ is the most abundant splice variant of gephyrin. Insertion of cassettes 4 and 5 (light gray boxes) generates the ge $(2,4,5)$ variant. The constructs are $\mathrm{N}$-terminally tagged either with Venus or mrfp. $B$, COS-7 cells cotransfected with vege(2), mrfpge(2), and the myc-tagged GlyR subunit GlyR $\mathrm{R}_{\alpha 1} \beta \mathrm{gb}$ form gephyrin clusters (both in the green and the red channels) that colocalize with surface $G_{l y R_{\alpha 1}} \beta g$ b immunostained $24 \mathrm{~h}$ after transfection. $C$, Over-expression of the truncated construct $\mathrm{mrfpG}(2)$ results in a diffuse distribution and a reduction in the size of gephyrin/Gly $\mathrm{R}_{\alpha 1} \beta \mathrm{gb}$ coclusters (arrows in the magnified images). Scale bar, $10 \mu \mathrm{m} . \boldsymbol{D}, \boldsymbol{E}$, Quantification of the size (D) and relative cellular surface area covered by vege(2)/Gly $\mathrm{R}_{\alpha 1} \beta \mathrm{gb}$ clusters $(\boldsymbol{E})$ in $\operatorname{COS}-7$ cells cotransfected with the different mrfptagged gephyrin constructs [means \pm SEM; $N=$ at least 10 cells for each condition; Student's $t$ test, significant differences from mrfpge(2) with $p \leq 10^{-3}\left(^{* *}\right), 10^{-2}(* *)$, and $\left.5 \times 10^{-2}(*)\right]$.

$60 \mathrm{NA}=1.45)$, a mercury lamp $(100 \mathrm{~W})$, and a heating chamber. Excitation and emission filters are as follows: D455/70 excitation and HQ605/20 emission for QD, D535/50 excitation and E590lp v2 emission for FM4-64 and mrfp, and HQ500/20 excitation and HQ535/30 for Venus (Chroma Technology). Consecutive frames (250 or 512) were acquired with an integration time of $75 \mathrm{~ms}$ with a charge-coupled device camera Cascade 512 (Roper Scientific) using Metaview (Molecular Devices; Roper Scientific). Single images of Venus- or mrfp-tagged constructs, as well as FM4-64, were taken before the recording. Recording sessions for each coverslip examined did not last $>30 \mathrm{~min}$.

Single-particle tracking. As described previously (Bonneau et al., 2005; Bannai et al., 2006; Ehrensperger et al., 2007), tracking of single QDs, which were identified by their fluorescence intermittence, was performed with MATLAB (MathWorks) using a homemade macro that accounts for blinking in the fluorescence signal. In brief, the method consists of two main steps, applied successively to each frame of the sequence. First, fluorescent spots are detected by cross-correlating the image with a Gaussian model of the point spread function. A least-squares Gaussian fit is applied (around the local maximum above a threshold) to determine the center of each spot with a spatial accuracy of 5-10 $\mathrm{nm}$ (depending on the signal-tonoise ratio). Second, QD trajectories are assembled automatically by linking, from frame to frame, the centers of fluorescent spots likely from the same QD. The association criterion is based on the assumption of free Brownian diffusion and takes into account short-blinking events. After completion of the process, a manual association step is performed, in which QD trajectories of maximal length are assembled from smaller fragments separated by longer blinking events that were not taken into account by the automatic linking procedure.

Quantitative analysis of diffusion coefficient. Analyses of mean square displacement (MSD) and initial diffusion coefficient (D) are reported in Bannai et al. (2006) and Ehrensperger et al. (2007). Briefly, physical parameters can be extracted from each trajectory $(x(\tau), y(\tau))$ by computing the MSD (Saxton and Jacobson, 1997), determined from the following formula:

$$
\begin{array}{r}
\operatorname{MSD}(n \tau)=\frac{1}{N-n} \sum_{i=1}^{N-n}\left[x((i+n) \tau-x(i \tau))^{2}\right. \\
+\left(y((i+n) \tau-y(i \tau))^{2}\right],
\end{array}
$$

where $\tau$ is the acquisition time and $N$ the total number of frames. This function enables the analysis of the lateral dynamics on short (initial diffusion coefficient) and long (types of motion) time scales. Different types of motion can be distinguished from the time dependence of the MSD (Saxton and Jacobson, 1997). For simple two-dimensional Brownian motion, the MSD $-n \tau$ plot is linear with a slope of $4 D$, where $D$ is the diffusion constant. If the MSD $-n \tau$ plot tends toward a constant value $L$, the diffusion is confined in a domain of size $\sim L$.

The initial $D$ was determined by fitting the initial 2 to 5 points of the MSD- $n \tau$ curve with $\operatorname{MSD}(n \tau)=4 D n \tau+b$. The cumulative probability $C(d)$ of $D$ defines the probability that $D$ is inferior to $d$.

To define gephyrin clusters and synaptic compartments (Dahan et al., 2003), vege(2) and FM4-64 images were filtered using the MIA interface running in MetaMorph (see above).

\section{Results}

We have studied the clustering and dynamic behavior of the most abundant and ubiquitous gephyrin variant (referred to as ge(2) or "wild-type" gephyrin). The strategy used to interfere with the oligomerization of ge(2) was based on the coexpression of a range of constructs able to interact either with the G or the E domain of the full-length protein. To this aim, we generated the full-length ge $(2,4,5)$ splice variant, the deletion mutants $G(2)$ and $G(2,5)$ that correspond to the isolated $G$ domains from ge(2) and ge $(2,4,5)$, respectively, as well as a construct corresponding to the E domain of gephyrin (Fig. $1 A$ ). Whereas $\mathrm{G}(2)$ trimerizes in vitro, 

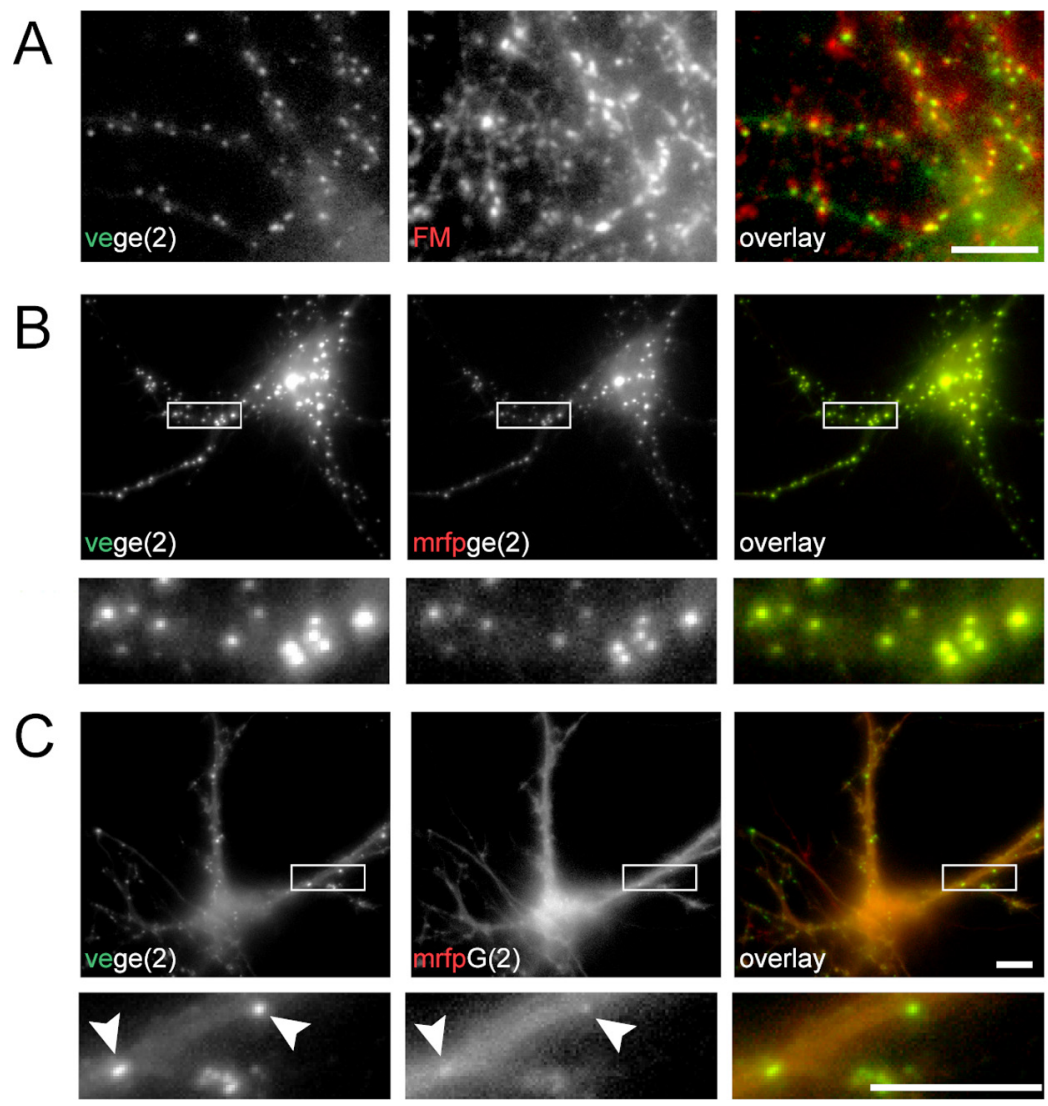

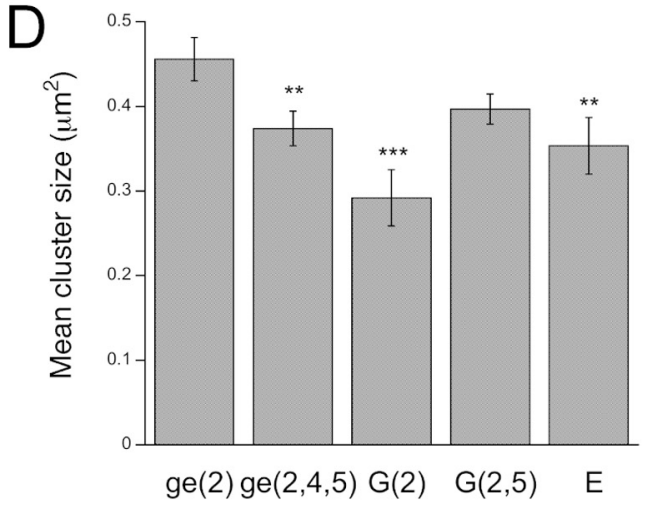

overlay
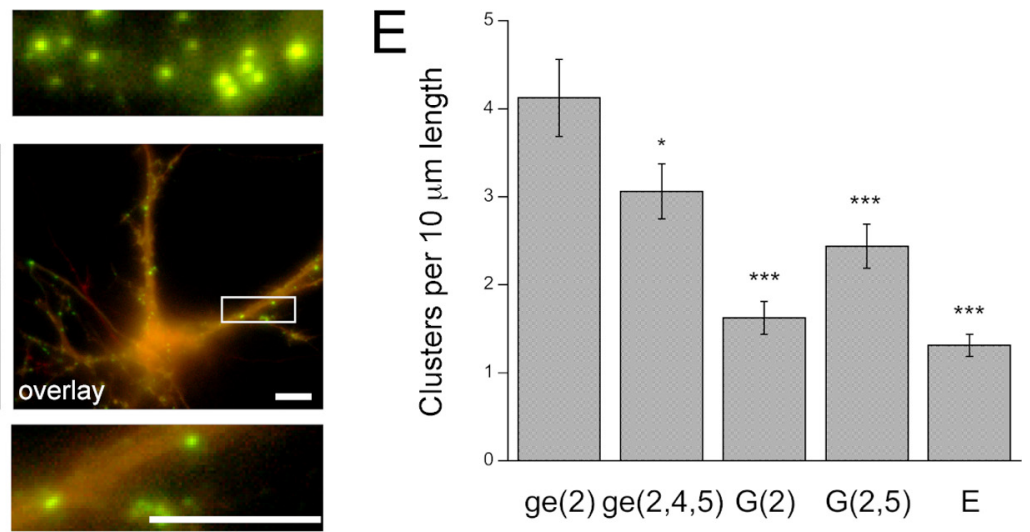

Figure 2. Expression pattern of gephyrin constructs in spinal cord neurons. $A$, Most of the dendritic vege(2) clusters colocalize with FM4-64-positive presynaptic terminals in live neurons (80.5 \pm $3.5 \%$, mean $\pm \mathrm{SEM}, n=13$ cells; $8-10 \mathrm{DIV}, 24-48 \mathrm{~h}$ after transfection). $\boldsymbol{B}$, Vege(2) and mrfpge(2) form clusters that colocalize in living neurons. $\boldsymbol{C}$, The truncated construct mrfpG(2) has a more diffuse distribution and causes a reduction in the size and number of gephyrin clusters (indicated by arrows). Magnified details are shown for each image. Scale bar, $10 \mu \mathrm{m}$. $\boldsymbol{D}, \boldsymbol{E}, \mathrm{Quantification} \mathrm{of}$ the size $(\boldsymbol{D})$ and the number of vege(2) clusters per length of dendrite $(\boldsymbol{E})$ in neurons cotransfected with the different mrfp-tagged gephyrin constructs [mean \pm SEM; $n=10$ cells per condition; Student's $t$ test, significant differences from mrfpge(2) with $p \leq 10^{-3}\left(^{* * *}\right), 10^{-2}\left({ }^{* *}\right)$, and $5 \times 10^{-2}\left(^{*}\right)$ ].

$\mathrm{G}(2,5)$ has been reported to form monomers or dimers (Bedet et al., 2006; Saiyed et al., 2007) (G. Schwarz, personal communication). Two sets of constructs were generated, tagged either with Venus (ve) or with mrfp at the $\mathrm{N}$ terminus.

Experiments were performed in COS-7 cells and 8-10 d old rat spinal cord primary neurons. COS-7 cells were chosen as model system, because this cell line expresses very low levels of endogenous gephyrin. Since COS-7 cells do not express GlyRs, we cotransfected them with a recombinant GlyR $\alpha 1$ subunit containing the gephyrin-binding sequence of the $\beta$ subunit (Meier et al., 2000a,b). This subunit, referred to as $\mathrm{GlyR}_{\alpha 1} \beta \mathrm{gb}$, forms functional homomeric receptors able to bind gephyrin.

\section{Interference with gephyrin clustering in COS-7 cells}

Venus-tagged full-length gephyrin [vege(2)] and myc-tagged GlyR $_{\alpha 1} \beta \mathrm{gb}$ were coexpressed in COS-7 cells together with the various mrfp-tagged constructs (Fig. 1; supplemental Fig. S1, available at www.jneurosci.org as supplemental material). Cells were fixed after $24 \mathrm{~h}$, after $\mathrm{GlyR}_{\alpha 1} \beta \mathrm{gb}$ surface immunolabeling. As expected, the full-length protein mrfpge(2) formed large clusters that colocalized with vege (2) and surface-labeled GlyR ${ }_{\alpha 1} \beta g b$ (Fig. $1 B$ ). We also observed gephyrin clusters that did not colocalize with surface receptors but could correspond to gephyrin bound to intracellular GlyR-containing vesicles as reported previously (Bedet et al., 2006; Hanus et al., 2006). In contrast, a rather diffuse mrfp signal was observed in cells transfected with mrfpG(2) (Fig. 1C). This construct formed few and small clusters associated with vege (2) and $\mathrm{GlyR}_{\alpha 1} \beta \mathrm{gb}$, suggesting an interaction of the truncated protein with full-length gephyrin below the cell surface. Furthermore, the presence of $\operatorname{mrfpG}(2)$ caused a noticeable reduction in the size of the full-length vege(2) clusters.

The average size and relative cellular surface covered by vege(2) clusters colocalizing with $\mathrm{GlyR}_{\alpha 1} \beta \mathrm{gb}$ were quantified after cotransfection with the different mrfp-tagged gephyrin mutants (Fig. 1D,E). All the tested constructs had a strong effect on gephyrin clusters size. The most dramatic change was found for the construct $G(2)$, which caused a $60 \%$ reduction of cluster size. We also found $30 \%$ reduction in gephyrin cluster size in COS-7 cells expressing the splice variant mrfpge $(2,4,5)$. Analogous results were obtained from comparing the relative area of the plasma membrane covered by gephyrin clusters in the presence of the different constructs (Fig. $1 E$ ). These data demonstrate that the ge $(2,4,5)$ variant and the isolated $G(2), G(2,5)$, and $E$ domains exert a dominant-negative effect on the clustering of wild-type gephyrin $[\mathrm{ge}(2)]$ in COS-7 cells.

Interference with gephyrin clustering in spinal cord neurons Changes in gephyrin cluster size and number were then investigated in neuronal cells. As in COS-7 cells, we expressed vege(2) together with the various mrfp constructs in spinal cord neurons cultured for 8-10 DIV (Fig. 2; supplemental Fig. S2, available at www.jneurosci.org as supplemental material). Under these conditions, the vast majority of the dendritic vege(2) clusters (80.5 \pm $3.5 \%$ ) colocalized with the presynaptic marker FM4-64 (Fig. 2A), 
A
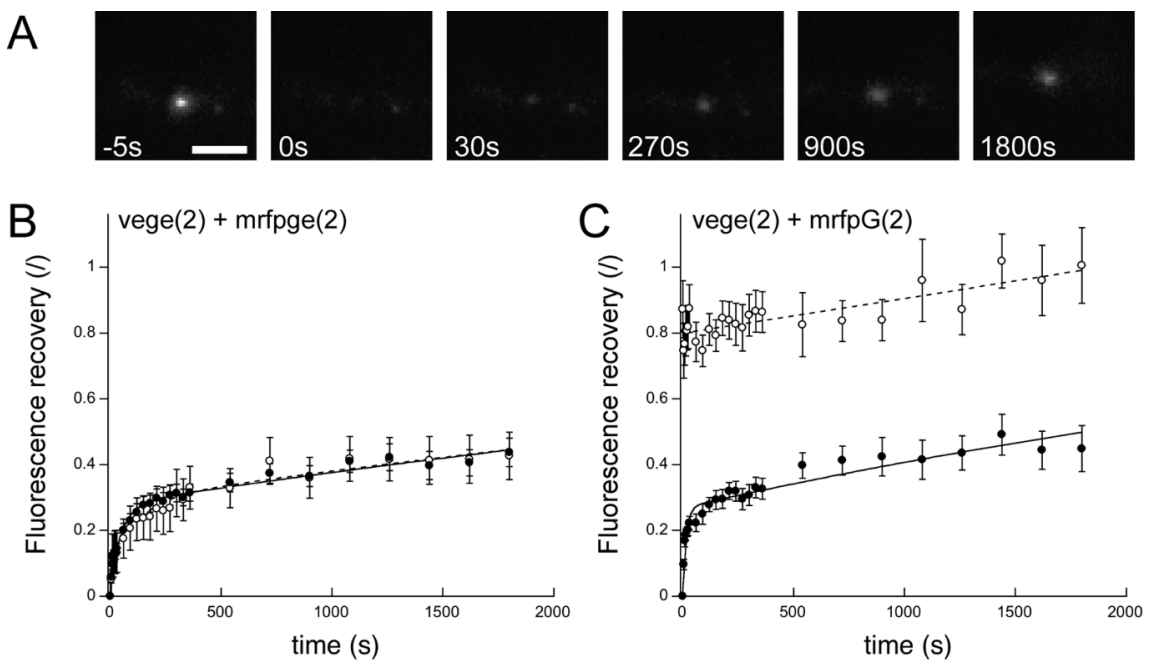

$\mathrm{D}$

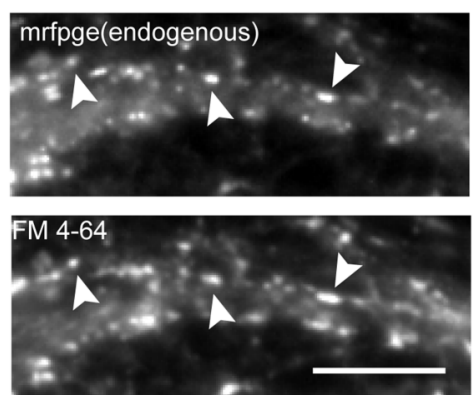

E

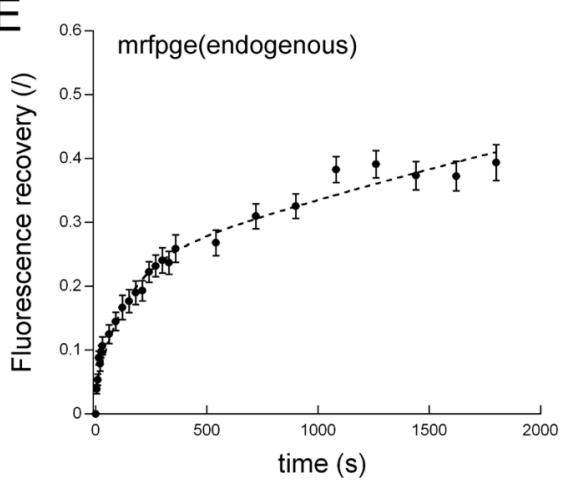

Figure 3. Dynamics of gephyrin clusters analyzed by FRAP. $A$, Fluorescence recovery of a gephyrin cluster after photobleaching in a living spinal cord neuron transfected with vege(2) and mrfpge(2). Scale bar, $3 \mu \mathrm{m}$. $\boldsymbol{B}$, Fluorescence recovery of dendritic gephyrin clusters in 8-10 DIV neurons after $24-48 \mathrm{~h}$ of expression of vege(2) and mrfpge(2). Venus and mrfp-positive puncta were bleached simultaneously, and the recovery of both constructs was recorded for $30 \mathrm{~min}$. Vege(2) (black circles, black line) and mrfpge(2) (white circles, dotted line) show identical times of recovery. $\boldsymbol{C}$, The recovery of the truncated construct $\operatorname{mrfpG}(2)$ (white circles, dotted line) is almost instantaneous in neurons cotransfected with vege(2) and $\operatorname{mrfp} G(2)$, whereas the recovery of vege(2) (black circles, black line) remains unchanged (compare with the black line shown in $\boldsymbol{B}$ and see supplemental Table S1, available at www.jneurosci.org as supplemental material). Fluorescence intensities (mean \pm SEM) were normalized to the value before bleaching and to the first value after the bleaching event [with the exception of $\operatorname{mrfpG}(2)$; for details, see Materials and Methods]. $N>15$ clusters from $\geq 5$ cells and $>3$ independent experiments per condition. $D$, Endogenous mrfp-gephyrin clusters in spinal cord neurons from a knock-in mouse model (top) colocalize with active presynaptic terminals (FM4-64 staining, bottom image, see arrowheads). Scale bar, $10 \mu \mathrm{m}$. $\boldsymbol{E}$, Fluorescence recovery of mrfp- gephyrin clusters in spinal cord neurons from knock-in animals (mean $\pm \mathrm{SEM} ; n=28$ clusters from 7 cells; DIV $10-11$ ). Note that the rate of recovery of endogenous gephyrin is very similar to that of the over-expressed protein (compare with the traces in $\boldsymbol{B}$ ).

in agreement with previous results on the colocalization of gephyrin with endogenous GlyR clusters or the vesicular transporter protein VIATT at inhibitory synapses (Dumoulin et al., 2000; Bedet et al., 2006; Hanus et al., 2006). We could, therefore, directly image synaptic gephyrin clusters in living neurons, avoiding fixation and immunolabeling of the receptor. Similar to our results obtained in COS-7 cells, mrfpge(2) formed clusters that colocalized with vege(2) (Fig. $2 B$ ), whereas the deletion mutant $\mathrm{mrfpG}(2)$ was distributed diffusely throughout the cytoplasm and rarely formed clusters (Fig. $2 C$ ). Moreover, the size and the number of the clusters formed by vege(2) in the presence of $\mathrm{mrfpG}(2)$ were noticeably reduced.

The size and number of vege(2) clusters was quantified in dendrites of neurons cotransfected with the different mrfptagged mutants (Fig. 2D,E). The most prominent effect was caused by $\operatorname{mrfpG}(2)$, which decreased the size and number of gephyrin clusters by 40 and $55 \%$, respectively. The deletion construct mrfpE had a similar influence on gephyrin clustering (20

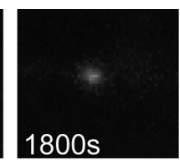

and $60 \%$ ), whereas the effects of $\mathrm{mr}$ fpG $(2,5)$ and mrfpge $(2,4,5)$ were less pronounced.

Together, these data demonstrate that the effects of the different deletion constructs on vege(2) cluster size are paralleled by changes in the number of clusters. The ability of $\operatorname{mrfpG}(2,5)$ to interfere with gephyrin clustering suggests that the presence of cassette 5 does not fully abolish the binding properties of the $G$ domain. Nonetheless, the effect observed for the ge $(2,4,5)$ variant may be related to a reduced propensity of the $G(2,5)$ domain to form trimers.

\section{Characterization of the steady-state dynamics of gephyrin clusters}

The dynamic properties of gephyrin [vege(2)] and the various mrfp constructs were analyzed using FRAP on dendritic gephyrin clusters in 8-10 DIV rat spinal cord neurons (Fig. 3; supplemental Fig. S3, available at www.jneurosci.org as supplemental material). The rate of fluorescence recovery provides a direct measure of how rapidly the tagged molecules replace bleached proteins in a cluster (Fig. $3 A$ ). Both Venus and mrfp were photobleached simultaneously to $\sim 20-30 \%$ of their initial intensity, and their recoveries were monitored using specific filters. For comparison, the recovery traces were generally normalized to the value before bleaching $(=1)$, the ongoing photobleaching of nonbleached puncta, and to the first point after photobleaching $(=0)$ (see Materials and Methods for details).

The time and the extent of recovery were nearly identical for vege(2) and mrfpge(2) (Fig. 3B), indicating that the dynamic properties of recovery are independent of the protein tag. The full-length protein has a relatively slow exchange rate of $\sim 40-45 \%$ within 30 min after bleaching, reflecting the oligomerization of gephyrin at inhibitory synapses. In the case of $\operatorname{mrfpG}(2)$, which barely accumulates at vege(2) clusters, the recovery was almost immediate and occurred during the dead time of the experiment (Fig. 3C). For this construct, fast diffusion seems to be the dominant factor accounting for the rapid fluorescence recovery. However, mr$\mathrm{fpG}(2)$ did not alter the exchange rate of the full-length protein, as the fluorescence recovery of vege(2) was comparable with that in the presence of mrfpge(2) (Fig. $3 B, C$ ). Similarly to $\mathrm{mrfpG}(2)$, mrfpge $(2,4,5)$ and the remaining mrfp constructs differed in their exchange rates but did not affect the recovery of vege(2) clusters (supplemental Fig. S3, supplemental Table S1, available at www.jneurosci.org as supplemental material).

The potential effects of overexpression on the observed exchange rates of gephyrin clusters were investigated by performing additional FRAP experiments on spinal cord primary neurons from an mRFP-gephyrin knock-in mouse model (P. Machado and A. Triller, unpublished observations). In these animals, 
mRFP-tagged gephyrin is expressed at endogenous levels, and it forms synaptic clusters in front of active presynaptic terminals (FM4-64 labeling) (Fig. 3D). The recovery rate of synaptic mrfp-gephyrin was similar to that of the over-expressed protein, reaching $\sim 40 \%$ within $30 \mathrm{~min}$ (Fig. 3E; supplemental Table $\mathrm{S} 1$, available at www.jneurosci.org as supplemental material). This indicates that the exchange rate of gephyrin depends on the properties of the gephyrin molecule rather than on its expression levels.

Together, our results show that gephyrin cluster formation is a dynamic and reversible process. Moreover, the various deletion constructs exchange faster than wild-type full-length gephyrin, consistent with their decreased ability to form intermolecular interactions. Finally, the constructs do not influence the dynamics of gephyrin clusters at steady state, although they can alter the size of the clusters.

\section{Changes in GlyR diffusion in spinal cord neurons}

Our FRAP experiments showed that the mutant gephyrin constructs did not affect the exchange rate of full-length gephyrin at inhibitory synapses. To understand this unexpected result, we analyzed the effect of the interfering constructs on the mobility of GlyRs by SPT. These experiments were performed in 8-10 DIV spinal cord neurons by immunochemically labeling the $\alpha$ subunit of endogenous GlyRs with QDs (Dahan et al., 2003). Since the emission wavelengths of FM4-64 and mrfp overlap, neurons were singly transfected with the Venus-tagged versions of ge(2), ge $(2,4,5), G(2), G(2,5)$, or E. The subcellular distribution of these constructs was indistinguishable from the mrfp-tagged constructs. Images of FM-stained synapses were filtered using a procedure that allows the simultaneous detection of small and large structures displaying low and high intensities, respectively. This approach was used to discriminate between trajectories of QDs that transit over and outside of synapses (Fig. $4 A$ ).

Within the time scale of the recordings ( $\sim 20 \mathrm{~s})$, endogenous GlyRs bound to QDs exhibited distinct behaviors in neurons. Depending on the length of time spent over or outside FMstained synapses, receptors could be classified into three types: synaptic, extrasynaptic, and swapping between the two compartments (Fig. $4 A$ ). In agreement with previous results (Dahan et al., 2003; Charrier et al., 2006; Ehrensperger et al., 2007), synaptic GlyRs displayed a confined mobility (Fig. $4 B$ ), whereas extrasynaptic receptor had the features characteristic of free Brownian diffusion (Fig. 4C), as indicated by the linear relationship between the MSD and time.

To explore to which extent the altered oligomerization properties of gephyrin could affect the lateral diffusion of GlyRs, we compared Ds obtained in the presence of the different constructs
(Fig. 4; supplemental Fig. S4, available at www.jneurosci.org as supplemental material). Diffusion coefficients were calculated using the initial points of the MSD over time for each QD. We compared cumulative probability distributions and median instead of mean values because D values were spread over four orders of magnitude.

The median D values obtained for synaptic and extrasynaptic GlyRs were consistent with previous data (Charrier et al., 2006). The analysis of synaptic trajectories of GlyRs yielded comparable values of $\mathrm{D}$ in the presence of the various constructs (Table 1, Fig. $4 D)$. However, transfection of ge $(2,4,5), \mathrm{G}(2)$, and E caused significantly different $\mathrm{D}$ values in the case of extrasynaptic GlyR (Table 1, Fig. 4E). The most marked influence was observed for the full-length gephyrin splice variant vege $(2,4,5)$, for which the median $\mathrm{D}$ was three times higher than for vege $(2)$. We observed a similar trend in the presence of $\mathrm{G}(2,5)$, although this did not reach significance.

Whereas we did not observe significant changes in the diffusion of synaptic GlyRs, a result consistent with our FRAP exper- 
iments, the lateral diffusion of extrasynaptic GlyRs was modified by the presence of the interfering constructs. These results not only support the notion that extrasynaptic GlyRs can diffuse in a gephyrin-bound state (Ehrensperger et al., 2007) but also demonstrate that the diffusion of extrasynaptic GlyR-gephyrin complexes can be modified by factors able to interfere with gephyrin oligomerization.

\section{Changes in GlyR diffusion in COS-7 cells}

COS-7 cells express low-level of endogenous gephyrin, and diffusion measurements obtained in these cells are independent of the width of the synaptic cleft ( $\sim 20 \mathrm{~nm})$, which might influence the mobility of receptors bound to QDs in neurons. Diffusion of transfected GlyR ${ }_{\alpha 1} \beta g$ b was, therefore, studied in COS-7 cells expressing $\mathrm{GlyR}_{\alpha 1} \beta \mathrm{gb}$ and vege(2) together with one of the various mrfp-tagged constructs. Binary images of vege(2) clusters were used as reference to classify the types of motion of the transfected receptor (Fig. $5 A, B$ ).

Although the various mrfp-tagged constructs did not significantly alter the diffusion coefficient of $\mathrm{GlyR}_{\alpha 1} \beta \mathrm{gb}$ over clusters (Fig. 5C; supplemental Fig. S5, available at www.jneurosci.org as supplemental material), the analysis of trajectories of $\mathrm{GlyR}_{\alpha 1} \beta \mathrm{gb}$ outside clusters revealed dramatic differences (Fig. 5D; supplemental Fig. S5, available at www.jneurosci.org as supplemental material). Compared with mrfpge(2), expression of all the other constructs leads to higher values of median D (supplemental Table S2, available at www.jneurosci.org as supplemental material). In particular, $\mathrm{GlyR}_{\alpha 1} \beta \mathrm{gb}$ showed a 3-4-fold increase of D outside clusters in the presence $\operatorname{mrfpG}(2)$ and $\operatorname{mrfpE}$ as compared with mrfpge(2). The mrfpge $(2,4,5)$ variant increased the median $\mathrm{D}$ of $\mathrm{GlyR}_{\alpha 1} \beta \mathrm{gb}$ by 1.5 fold. In accordance with the observation that at least $40 \%$ of the receptor diffuses outside clusters in a gephyrin-bound state (Ehrensperger et al., 2007), more than 10-fold higher median D values were found in COS-7 cells expressing GlyR $_{\alpha 1} \beta g b$ only.

The stronger effects of the different constructs on the mobility of GlyRs in COS-7 cells may have several reasons. GlyR ${ }_{\alpha 1} \beta g b$ in COS-7 cells forms homopentamers, whereas the endogenous receptor in mature neurons is believed to have a stoichiometry of three $\beta$ and two $\alpha 1$ subunits (Grudzinska et al., 2005). Since only the $\beta$ subunit has the gephyrin-binding motif, the number of gephyrin molecules bound to the extrasynaptic receptor is expected to be larger in COS-7 cells than in neurons. The influence of the constructs might, therefore, be more pronounced in COS-7 cells. Moreover, a number of trajectories of QDs from nontransfected neurons may have been included in the analysis, contributing to a reduction in the difference between the various D curves.

Nonetheless, the experiments performed in COS-7 cells fully confirm the results obtained in neurons. The lateral diffusion of $\mathrm{GlyR}_{\alpha 1} \beta \mathrm{gb}$ outside of gephyrin clusters can be accurately modulated according to the oligomerization state of gephyrin, similarly to what had been observed for extrasynaptic GlyRs.

\section{Discussion}

Dynamic equilibrium of GlyR-gephyrin clusters: structural stability without loss of plasticity

In the absence of GlyRs in non-neuronal cells, gephyrin ( $S$ for scaffold) is distributed diffusely throughout the cytoplasm (Kirsch et al., 1995; Hanus et al., 2006). Similarly, exogenous GlyRs alone ( $R$ for receptor) have a diffuse pattern on the plasmamembrane (Kirsch et al., 1995; Meier et al., 2000a). When both gephyrin and GlyRs are present, they form coclusters $\left((R S)_{n}\right)$ at the plasmamembrane (Meier et al., 2000a; Hanus et al., 2006). The
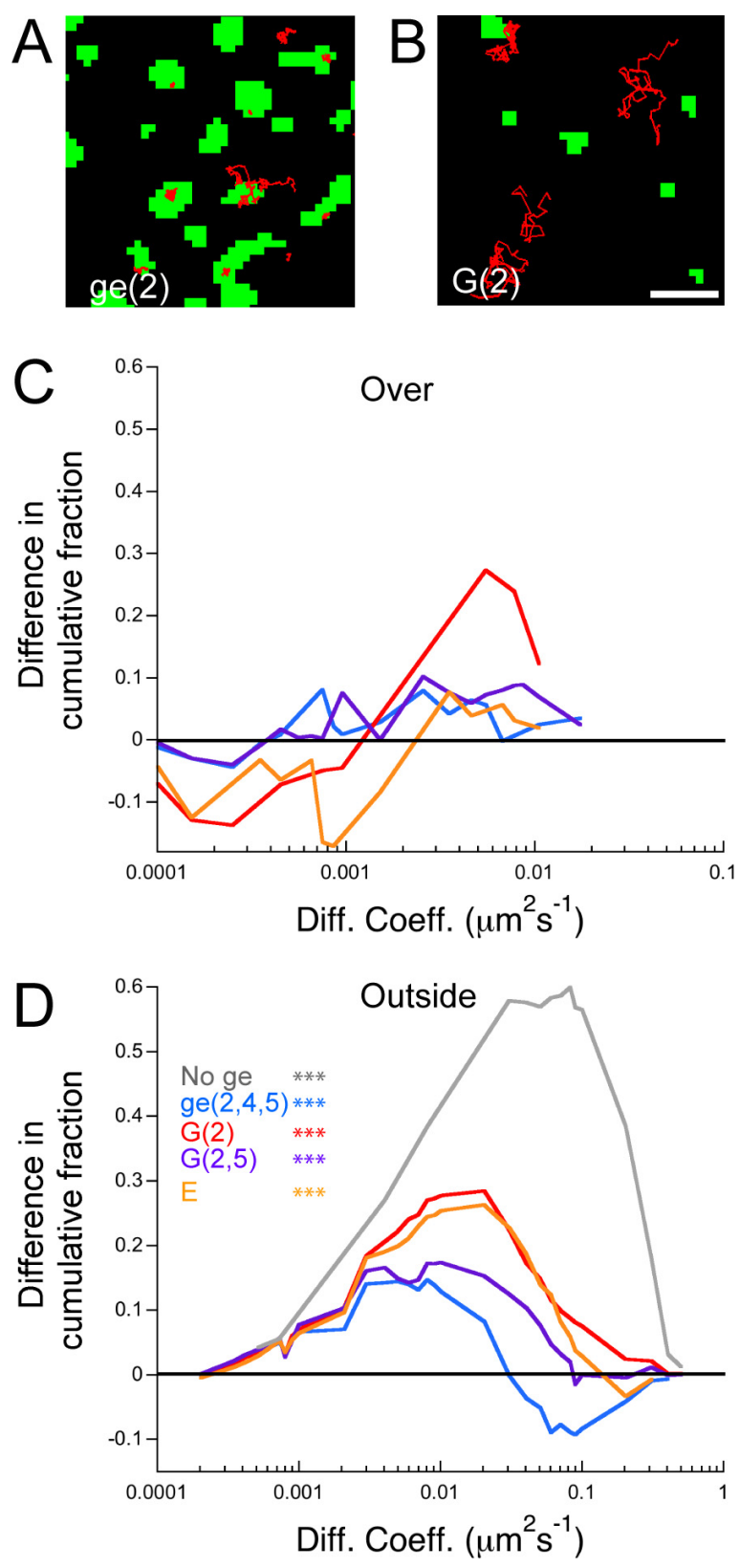

Figure 5. GlyR diffusion in COS-7 cells transfected with the various constructs. $A, B$, Trajectories of QD-coupled GlyR $\mathrm{R}_{\alpha 1} \beta \mathrm{gb}$ (red) superimposed to binary images of vege(2) clusters (green) in COS-7 cells cotransfected with GlyR ${ }_{\alpha 1} \beta g$ b and vege(2) together with mrfpge(2) (A) or mrfpG(2) (B). Scale bar, $2.5 \mu \mathrm{m}$. C, D, Diffusion coefficients of QD-coupled $G_{1 y} R_{\alpha 1} \beta g b$ were calculated from trajectories located over $(\boldsymbol{C})$ and outside of $(\boldsymbol{D})$ vege(2) clusters in COS-7 cells expressing the different mrfp-tagged constructs. Differences in the cumulative fraction of diffusion coefficients relative to mrfpge(2)-expressing cells show an increased mobility of receptors in the presence of all tested constructs [ge $(2,4,5)$, blue; $G(2)$, red; $G(2,5)$, purple; $E$, orange; or in the absence of gephyrin, gray]. Kolmogorov-Smirnov test: significant differences from vege(2) with $p \leq 10^{-3}{ }^{* * *}$ ) (see supplemental Table S2, available at www.jneurosci.org as supplemental material).

reciprocal stabilization of GlyRs and gephyrin is thus needed for the formation of clusters that mimic the structure of glycinergic postsynaptic scaffolds. As shown previously, a significant fraction of GlyRs outside of these clusters is also bound to gephyrin molecules $(R S)$ (Ehrensperger et al., 2007). Therefore, receptor and scaffold proteins exchange between different states that may be described by the following dynamic equilibrium: 


$$
R+S \stackrel{1}{\leftrightarrows} R S \stackrel{2}{\leftrightarrows}(R S)_{n}
$$

This dynamic equilibrium is based on the binding between gephyrin and the receptor (equilibrium 1), as well as on gephyrin-gephyrin interactions (equilibrium 2). In our SPT and FRAP experiments in neurons, we directly observed the dynamic exchange of GlyRs and gephyrin, respectively, between the synaptic $\left((R S)_{\mathrm{n}}\right)$ and extrasynaptic states $(R S$ and $R+S)$. To discriminate between the $R S$ and $R$ states, we have examined the diffusion of GlyRs in COS-7 cells in the absence of gephyrin. From these data, we could estimate that the majority of the nonclustered GlyRs are bound to gephyrin.

We then challenged the receptor-scaffold equilibrium by expressing constructs that interfere with gephyrin oligomerization. The over-expression of the isolated G or E domains of gephyrin in neurons caused a reduction of clustered gephyrin. Since these deletion constructs have fewer oligomerization sites than the fulllength protein, they are likely to saturate gephyrin-binding sites and thus block gephyrin-gephyrin interactions (see below). As a consequence, the deletion constructs stabilize GlyR-gephyrin complexes in the $R S$ state by inhibiting the association of GlyRgephyrin complexes with existing gephyrin clusters.

It could be expected that an interference with the clustering of gephyrin would be reflected in an altered exchange rate between synaptic and extrasynaptic gephyrin pools. However, our FRAP experiments of synaptic full-length gephyrin revealed that the exchange of gephyrin was not affected by the presence of isolated $\mathrm{G}$ or $\mathrm{E}$ domains. Although the deletion constructs themselves exchanged almost instantaneously, they did not modulate the recovery of clustered full-length gephyrin. This finding suggests that full-length gephyrin and the different constructs had reached a steady state by the time the experiments were performed (24-48 $\mathrm{h}$ after transfection). In other words, the remaining gephyrin clusters were those "allowed" under the given conditions and were mostly composed of the full-length (Venustagged) gephyrin, as judged by the low abundance of mrfpfluorescence within the clusters. It is possible that the $\mathrm{G}$ and $\mathrm{E}$ constructs bind to the periphery of the clusters while being excluded from their innermost part. In contrast, an acute disruption of gephyrin-gephyrin binding would be predicted to result in the disassembly of gephyrin clusters along with a (transiently) slower recovery rate, until a new equilibrium is reached.

According to this interpretation, the size of synaptic gephyrin clusters can be dynamically regulated through the modulation of gephyrin-gephyrin interactions. As a consequence, the variation of the number of synaptic-binding sites may lead to changes in the amount of GlyRs at synapses, as, for example, seen in response to altered excitatory synaptic activity (Lévi et al., 2008). Thus, while providing structural stability for the clustering of receptors, gephyrin scaffolds undergo continuous assembly and disassembly that may be related to the expression of synaptic plasticity.

\section{Gephyrin clustering and GlyR diffusion}

We have analyzed the diffusion properties of endogenous GlyRs in the presence of isolated G or E domains of gephyrin by SPT in spinal cord neurons. These neurons also express GABA receptors, which accumulate at gephyrin-containing postsynaptic differentiations (Bohlhalter et al., 1994). However, the interaction of gephyrin with different GABA receptor subtypes is not fully understood (Kneussel et al., 2001; Lévi et al., 2004; Tretter et al.,
2008). For this reason, we have focused on the diffusion of GlyRs as a read-out of the receptor-gephyrin interaction, which has been very well characterized (Meyer et al., 1995; Schrader et al., 2004). The mobility of the extrasynaptic pool of GlyRs was notably increased in the presence of the gephyrin deletion constructs, confirming the existence of extrasynaptic GlyR-gephyrin complexes that depend on the oligomerization of gephyrin. In contrast, the diffusion of synaptic GlyRs did not differ significantly in the presence of the deletion constructs. This finding is in agreement with our FRAP data, suggesting that the gephyrin clusters that remain under these conditions are mostly composed of the full-length protein (see above).

Although increasing the mobility of extrasynaptic GlyRgephyrin complexes, the $\mathrm{G}$ or E domains decreased the size and number of gephyrin clusters at synapses (Table 2). To test whether these effects are related, we compared the mean cluster size and the diffusion coefficient of $\mathrm{GlyR}_{\alpha 1 \beta \mathrm{gb}}$ in the presence of the different deletion constructs in COS-7 cells. The advantage of this cellular model is that it lacks some of the constraints present in neurons, namely synaptic connections and endogenous gephyrin splice variants. Furthermore, the levels of the coexpressed proteins in COS-7 cells are comparable, whereas in neurons the overexpression of an interfering protein exceeds the endogenous gephyrin and receptor levels by far. Our analysis in COS-7 cells revealed a significant and inverse correlation between the size of gephyrin clusters and the diffusion of the GlyR $_{\alpha 1 \beta \mathrm{gb}}$ outside of clusters (Fig. $6 A$ ).

Several explanations may account for the increased mobility of the extrasynaptic receptors in the presence of the deletion constructs. For instance, whenever a gephyrin molecule (bound to the GlyR) is blocked from further oligomerization by isolated G domains, its potential to interact with other gephyrin molecules is reduced, as is the size of the GlyR-gephyrin complex (Fig. 6B). As a consequence, the mobility of the complex would be increased, particularly when taking into account the crowded cytoplasmic environment. Additionally, specific interactions such as those between the GlyR-gephyrin complex and the cytoskeleton may contribute to slowing down the receptor (Charrier et al., 2006; Hanus et al., 2006; Maas et al., 2006). The higher diffusion coefficients of extrasynaptic receptor complexes are thus likely to result not only from their lower molecular size but also from the reduced number of binding sites available for specific interactions with other proteins.

Based on this interpretation, we hypothesized that the observed correlation between the diffusion of GlyR-gephyrin complexes and gephyrin cluster size is related to the number of blocked gephyrin-binding sites by the interfering constructs (Fig. $6 B)$. We have, therefore, estimated the number of sites blocked by the different gephyrin constructs according to the available information on the oligomerization properties of gephyrin (for review, see Fritschy et al., 2008). Assuming the trimerization and the dimerization of the $\mathrm{G}$ and $\mathrm{E}$ domains, respectively (Schwarz et al., 2001; Sola et al., 2004; Kim et al., 2006), we found an inverse correlation between the estimated number of blocked binding sites of the interfering constructs and the diffusion of the GlyR $_{\alpha 1 \beta \mathrm{gb}}$ in COS-7 cells (Fig. 6C). It is noteworthy that this analysis does not take into account the binding affinities of the different constructs. Nonetheless, our model is independent from the exact oligomerization properties of the gephyrin domains and serves to illustrate the relative efficacy of different interfering constructs on gephyrin clustering. 


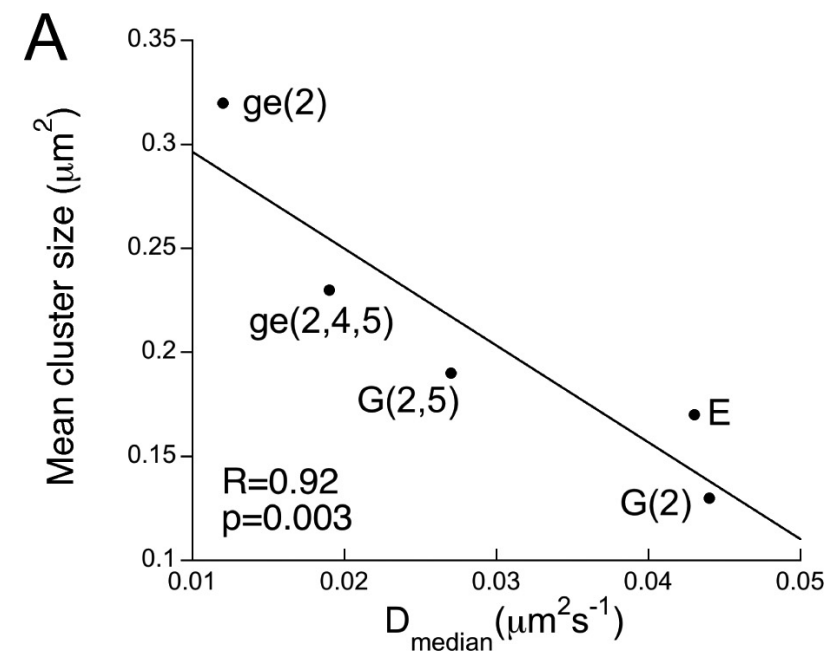

B
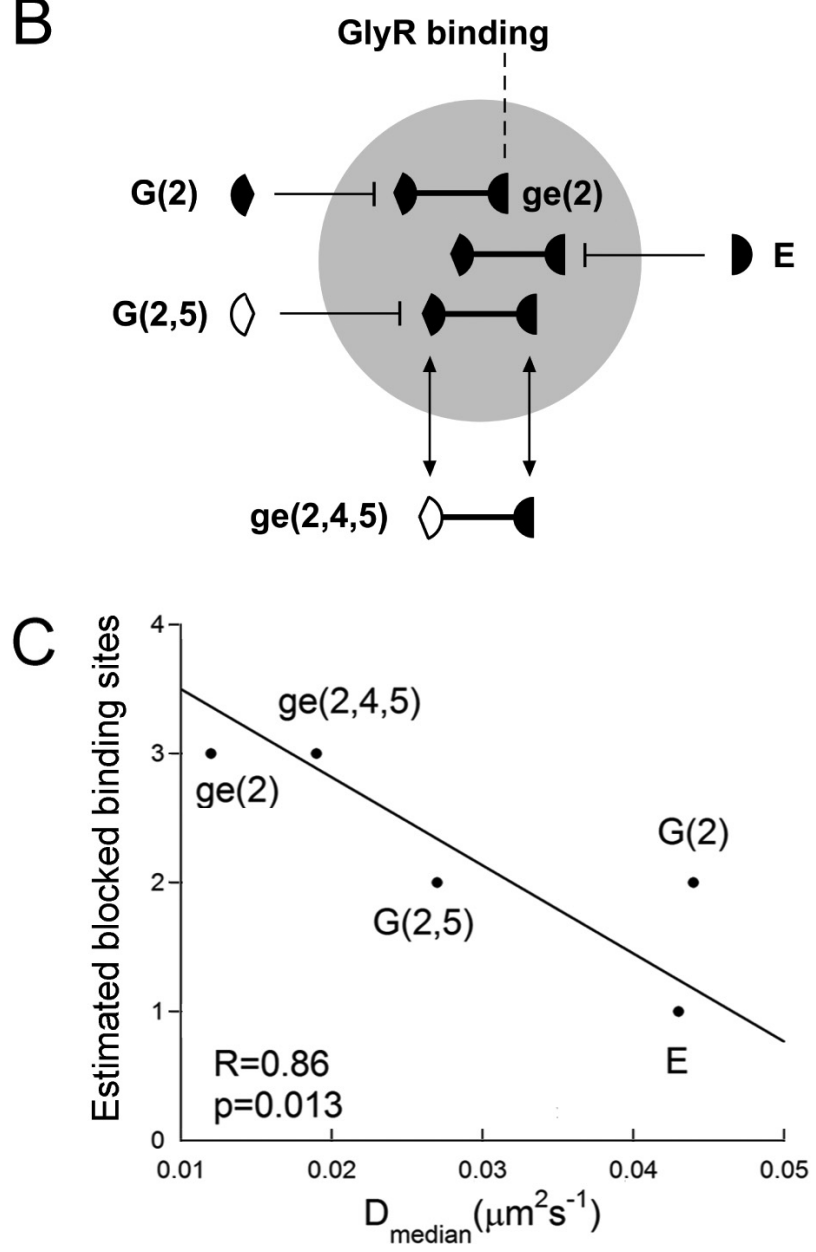

Figure 6. Modification of the gephyrin oligomerization state. $\boldsymbol{A}$, The reduction of gephyrin cluster size in COS-7 cells in the presence of different interfering constructs is paralleled by an increase in the diffusion of $\mathrm{GlyR}_{\alpha 1} \beta g b$ - gephyrin complexes outside of gephyrin clusters. This correlation between receptor diffusion coefficients and cluster size was also observed in neurons (data not shown). $\boldsymbol{B}$, Schematic representation of the predicted sites of action of the gephyrin constructs used in this study on gephyrin oligomers (synaptic or extrasynaptic) composed of full-length ge(2). Whereas isolated $\mathrm{G}$ or $\mathrm{E}$ domains interfere with gephyrin oligomerization by saturating binding sites, the full-length ge $(2,4,5)$ variant may replace ge(2) despite its reduced tendency to oligomerize. $\boldsymbol{C}$, An inverse correlation exists between the diffusion coefficients of GlyR ${ }_{\alpha 1} \beta g$ b outside gephyrin clusters in COS-7 cells and the estimated number of blocked binding sites of the interfering constructs, which appears to define their efficacy.
Modulation of synaptic organization by ge $(2,4,5)$

The full-length gephyrin variant containing cassette 5 [ge $(2,4,5)$ ] is present in the brain and spinal cord neurons both in rodents and humans (Meier et al., 2000b; Ramming et al., 2000; DavidWatine, 2001). Gephyrin variants containing cassette 5 have a decreased propensity to trimerize through the G domain (Bedet et al., 2006; Saiyed et al., 2007) and may tune the dynamic properties of bound GlyRs. In our experiments, the over-expression of ge $(2,4,5)$ in neurons caused a reduced clustering of synaptic gephyrin, accompanied by an increase in the mobility of extrasynaptic GlyRs (Table 2), similarly to isolated $\mathrm{G}$ and $\mathrm{E}$ domains. The lower propensity of ge $(2,4,5)$ to oligomerize is sufficient to explain the higher diffusion coefficient of the associated GlyR. However, whereas isolated G and E domains are likely to block gephyrin clustering through the saturation of binding sites, the ge $(2,4,5)$ variant can replace full-length gephyrin [ge(2)], albeit with a reduced tendency of oligomerization (Fig. $6 B$ ).

The expression of the $\mathrm{G}$ domain from cassette 5-containing gephyrin $[G(2,5)]$ yields further information on the oligomerization properties of the variant. $G(2,5)$ has an influence comparable with that of full-length ge $(2,4,5)$ on gephyrin clustering and GlyR diffusion. The effect of $G(2,5)$ was, however, less marked than that of the isolated $\mathrm{G}$ domain without cassette 5 , showing that the insertion of cassette 5 reduces, but does not abolish, the intermolecular interactions with the $\mathrm{G}$ domain of wild-type gephyrin. Since isolated G(2,5) domains were found to be mostly monomeric with a low proportion of dimers (Bedet et al., 2006; Saiyed et al., 2007) (G. Schwarz, personal communication), the heteromeric interaction between $\mathrm{G}$ and $\mathrm{G}(2,5)$ might indeed be more stable than $\mathrm{G}(2,5)$ homomers.

In conclusion, the number of receptors determines the synaptic strength and depends on the mobility of the receptor and its interaction with the synaptic scaffold (Triller and Choquet, 2005, 2008). Gephyrin variants containing cassette 5 alter both of these parameters, by increasing the lateral diffusion of extrasynaptic GlyRs and reducing the size of synaptic gephyrin clusters. Since endogenous gephyrin variants containing cassette 5 are expressed at relatively low levels (Paarmann et al., 2006b), fluctuations in ge $(2,4,5)$ expression may modulate the equilibrium between free and clustered states of the GlyR under physiological conditions.

\section{References}

Bannai H, Lévi S, Schweizer C, Dahan M, Triller A (2006) Imaging the lateral diffusion of membrane molecules with quantum dots. Nat Protoc 1:2628-2634.

Bedet C, Bruusgaard JC, Vergo S, Groth-Pedersen L, Eimer S, Triller A, Vannier C (2006) Regulation of gephyrin assembly and glycine receptor synaptic stability. J Biol Chem 281:30046-30056.

Bohlhalter S, Mohler H, Fritschy JM (1994) Inhibitory neurotransmission in rat spinal cord: co-localization of glycine- and GABAAreceptors at GABAergic synaptic contacts demonstrated by triple immunofluorescence staining. Brain Res 642:59-69.

Bonneau S, Dahan M, Cohen LD (2005) Single quantum dot tracking based on perceptual grouping using minimal paths in a spatiotemporal volume. IEEE Trans Image Process 14:1384-1395.

Charrier C, Ehrensperger MV, Dahan M, Lévi S, Triller A (2006) Cytoskeleton regulation of glycine receptor number at synapses and diffusion in the plasma membrane. J Neurosci 26:8502-8511.

Dahan M, Lévi S, Luccardini C, Rostaing P, Riveau B, Triller A (2003) Diffusion dynamics of glycine receptors revealed by single-quantum dot tracking. Science 302:442-445.

David-Watine B (2001) The human gephyrin (GPHN) gene: structure, chromosome localization and expression in non-neuronal cells. Gene 271:239-245.

Dumoulin A, Lévi S, Riveau B, Gasnier B, Triller A (2000) Formation of 
mixed glycine and GABAergic synapses in cultured spinal cord neurons. Eur J Neurosci 12:3883-3892.

Ehrensperger MV, Hanus C, Vannier C, Triller A, Dahan M (2007) Multiple association states between glycine receptors and gephyrin identified by SPT analysis. Biophys J 92:3706-3718.

Fritschy JM, Harvey RJ, Schwarz G (2008) Gephyrin: where do we stand, where do we go? Trends Neurosci 31:257-264.

Grudzinska J, Schemm R, Haeger S, Nicke A, Schmalzing G, Betz H, Laube B (2005) The beta subunit determines the ligand binding properties of synaptic glycine receptors. Neuron 45:727-739.

Hanus C, Ehrensperger MV, Triller A (2006) Activity-dependent movements of postsynaptic scaffolds at inhibitory synapses. J Neurosci 26:4586-4595.

Kim EY, Schrader N, Smolinsky B, Bedet C, Vannier C, Schwarz G, Schindelin H (2006) Deciphering the structural framework of glycine receptor anchoring by gephyrin. EMBO J 25:1385-1395.

Kirsch J, Wolters I, Triller A, Betz H (1993) Gephyrin antisense oligonucleotides prevent glycine receptor clustering in spinal neurons. Nature 366:745-748.

Kirsch J, Kuhse J, Betz H (1995) Targeting of glycine receptor subunits to gephyrin-rich domains in transfected human embryonic kidney cells. Mol Cell Neurosci 6:450-461.

Kneussel M, Betz H (2000) Clustering of inhibitory neurotransmitter receptors at developing postsynaptic sites: the membrane activation model. Trends Neurosci 23:429-435.

Kneussel M, Brandstätter JH, Gasnier B, Feng G, Sanes JR, Betz H (2001) Gephyrin-independent clustering of postsynaptic GABA(A) receptor subtypes. Mol Cell Neurosci 17:973-982.

Lardi-Studler B, Smolinsky B, Petitjean CM, Koenig F, Sidler C, Meier JC, Fritschy JM, Schwarz G (2007) Vertebrate-specific sequences in the gephyrin E-domain regulate cytosolic aggregation and postsynaptic clustering. J Cell Sci 120:1371-1382.

Lévi S, Logan SM, Tovar KR, Craig AM (2004) Gephyrin is critical for glycine receptor clustering but not for the formation of functional GABAergic synapses in hippocampal neurons. J Neurosci 24:207-217.

Lévi S, Schweizer C, Bannai H, Pascual O, Charrier C, Triller A (2008) Homeostatic regulation of synaptic GlyR numbers driven by lateral diffusion. Neuron 59:261-273.

Maas C, Tagnaouti N, Loebrich S, Behrend B, Lappe-Siefke C, Kneussel M (2006) Neuronal cotransport of glycine receptor and the scaffold protein gephyrin. J Cell Biol 172:441-451.

Meier J, Meunier-Durmort C, Forest C, Triller A, Vannier C (2000a) Formation of glycine receptor clusters and their accumulation at synapses. J Cell Sci 113:2783-2795.

Meier J, De Chaldée M, Triller A, Vannier C (2000b) Functional heterogeneity of gephyrins. Mol Cell Neurosci 16:566-577.

Meier J, Vannier C, Sergé A, Triller A, Choquet D (2001) Fast and reversible trapping of surface glycine receptors by gephyrin. Nat Neurosci 4:253-260.

Meyer G, Kirsch J, Betz H, Langosch D (1995) Identification of a gephyrin binding motif on the glycine receptor beta subunit. Neuron 15:563-572.
Paarmann I, Saiyed T, Schmitt B, Betz H (2006a) Gephyrin: does splicing affect its function? Biochem Soc Trans 34:45-47.

Paarmann I, Schmitt B, Meyer B, Karas M, Betz H (2006b) Mass spectrometric analysis of glycine receptor-associated gephyrin splice variants. J Biol Chem 281:34918-34925.

Prior P, Schmitt B, Grenningloh G, Pribilla I, Multhaup G, Beyreuther K, Maulet Y, Werner P, Langosch D, Kirsch J, Betz H (1992) Primary structure and alternative splice variants of gephyrin, a putative glycine receptor-tubulin linker protein. Neuron 8:1161-1170.

Ramming M, Kins S, Werner N, Hermann A, Betz H, Kirsch J (2000) Diversity and phylogeny of gephyrin: tissue-specific splice variants, gene structure, and sequence similarities to molybdenum cofactor-synthesizing and cytoskeleton-associated proteins. Proc Natl Acad Sci USA 97: 10266-10271.

Rosenberg M, Meier J, Triller A, Vannier C (2001) Dynamics of glycine receptor insertion in the neuronal plasma membrane. J Neurosci 21:5036-5044.

Saiyed T, Paarmann I, Schmitt B, Haeger S, Sola M, Schmalzing G, Weissenhorn W, Betz H (2007) Molecular basis of gephyrin clustering at inhibitory synapses: Role of G- and E-domain interactions. J Biol Chem 282:5625-5632.

Saxton MJ, Jacobson K (1997) Single-particle tracking: applications to membrane dynamics. Annu Rev Biophys Biomol Struct 26:373-399.

Schrader N, Kim EY, Winking J, Paulukat J, Schindelin H, Schwarz G (2004) Biochemical characterization of the high affinity binding between the glycine receptor and gephyrin. J Biol Chem 279:18733-18741.

Schwarz G, Schrader N, Mendel RR, Hecht HJ, Schindelin H (2001) Crystal structures of human gephyrin and plant $\mathrm{Cnxl}$ G domains: comparative analysis and functional implications. J Mol Biol 312:405-418.

Sola M, Bavro VN, Timmins J, Franz T, Ricard-Blum S, Schoehn G, Ruigrok RW, Paarmann I, Saiyed T, O'Sullivan GA, Schmitt B, Betz H, Weissenhorn W (2004) Structural basis of dynamic glycine receptor clustering by gephyrin. EMBO J 23:2510-2519.

Specht CG, Triller A (2008) Dynamics of synaptic scaffolds. Bioessays 30:1062-1074.

Théry M, Racine V, Pépin A, Piel M, Chen Y, Sibarita JB, Bornens M (2005) The extracellular matrix guides the orientation of the cell division axis. Nat Cell Biol 7:947-953.

Tretter V, Jacob TC, Mukherjee J, Fritschy JM, Pangalos MN, Moss SJ (2008) The clustering of $\mathrm{GABA}_{\mathrm{A}}$ receptor subtypes at inhibitory synapses is facilitated via the direct binding of receptor $\alpha 2$ subunits to gephyrin. J Neurosci 28:1356-1365.

Triller A, Choquet D (2005) Surface trafficking of receptors between synaptic and extrasynaptic membranes: and yet they do move! Trends Neurosci 28:133-139.

Triller A, Choquet D (2008) New concepts in synaptic biology derived from single-molecule imaging. Neuron 59:359-374.

Xiang S, Nichols J, Rajagopalan KV, Schindelin H (2001) The crystal structure of Escherichia coli MoeA and its relationship to the multifunctional protein gephyrin. Structure 9:299-310. 